\title{
Meta-analysis of aspirin-guided therapy of colorectal cancer
}

\author{
Johanna C. Mädge ${ }^{1}\left[\right.$ - Andreas Stallmach ${ }^{2} \cdot$ Lisa Kleebusch $^{3} \cdot$ Peter Schlattmann $^{1}$
}

Received: 26 October 2021 / Accepted: 3 February 2022 / Published online: 16 February 2022

(c) The Author(s) 2022

\begin{abstract}
Purpose colorectal cancer (CRC) is one of the most commonly diagnosed cancers worldwide. Some evidence has shown that aspirin can reduce the morbidity and mortality of CRC. The aim of this meta-analysis was to compare standard care of patients with CRC and standard care with the addition of aspirin in terms of the survival benefit.

Methods The systematic search was conducted by two independent reviewers in the databases PubMed and Web of Science. Survival data were extracted from studies published before July 2019. We searched for randomised controlled trials, cohort studies and case-control studies.

Results We included 27 studies in our meta-analysis. There was a sample size of 237,245 patients overall. Aspirin use after diagnosis was associated with an improvement in CRC-specific survival with a hazard ratio (HR) for cancer-related death of 0.74 (95\% CI: 0.62-0.89). Our analysis of overall survival data revealed reduced mortality with an HR of 0.82 (95\% CI: 0.74-0.90). Patients with the phosphatidylinositol-4, 5-bisphosphate 3-kinase catalytic subunit alpha (PIK3CA) mutation profited from postdiagnosis aspirin use $(\mathrm{HR}=0.74,95 \% \mathrm{CI}$ : $0.56-0.97)$. For a high expression of prostaglandin-endoperoxide synthase 2 (PTGS2) = COX-2, we found an HR of 0.65 (95\% CI: 0.52-0.82).

Conclusion Aspirin can improve the outcome of patients with CRC. PIK3CA mutation status and high expression of PTGS2 are associated with longer survival. However, randomised controlled trials are needed to investigate postdiagnosis aspirin use in CRC patients taking into account cancer stage and gene expression.
\end{abstract}

Keywords Aspirin $\cdot$ Colorectal cancer $\cdot$ Survival $\cdot$ Mortality $\cdot$ PIK3CA $\cdot$ PTGS2

\section{Abbreviations}

n.a

CRC

$\mathrm{CC}$

$\mathrm{RC}$

Pre

Post

PIK3CA

KRAS
Not available

Colorectal cancer

Colon cancer

Rectal cancer

Prediagnosis aspirin use

Postdiagnosis aspirin use

Phosphatidylinositol-4, 5-bispho-

sphate 3-kinase catalytic subunit

alpha

Kirsten rat sarcoma viral oncogene
BRAF

PTGS2

HLA class 1

CIMP

LINE-1

Phosphorylated AKT

CD274

\section{Introduction}

Johannamaedge@t-online.de

1 Department of Medical Statistics, Computer Sciences and Data Sciences, Jena University Hospital, 07743 Jena, Germany

2 Department of Internal Medicine IV, Jena University Hospital, 07743 Jena, Germany

3 Department of Internal Medicine, Thüringen-Kliniken Pößneck, 07381 Pößneck, Germany
V-raf murine sarcoma viral oncogene homolog B

Prostaglandin-endoperoxide synthase 2

Human leukocyte antigen class 1 $\mathrm{CpG}$ island methylator phenotype Long interspersed nuclear element Phosphorylated protein kinase B Cluster of differentiation 274 (=programmed cell death 1 ligand 1

Colorectal cancer (CRC) is one of the most prevalent cancer types around the world. Every year, about 945,000 people are diagnosed - 492,000 fatally (Weitz et al. 2005). Due to the high mortality, research into new methods of therapy should be increased. It is still unclear and controversially debated whether nonsteroidal anti-inflammatory drugs 
(NSAIDS) - acetylsalicylic acid (aspirin) in particularhave an influence on the development of CRC and whether they could be used for primary prevention of CRC (Bosetti et al. 2012). A recent meta-analysis (Haykal et al. 2019) did not find a reduction of cancer-related mortality and no reduced incidence of CRC. In recent years, randomised controlled trials have been used to investigate whether aspirin can help as an additional therapy after a diagnosis of CRC and to show whether patients have better outcomes than those receiving standard therapy (Michel et al. 2018). It is unclear whether the outcome depends on the time of starting aspirin, i.e., comparing patients who were taking aspirin before their diagnosis for other reasons (e.g., cardiovascular risk factors) and continued afterwards (primary prevention of CRC combined with tertiary prevention) with those who only began taking aspirin after their diagnosis of CRC (tertiary prevention). Furthermore, it is not clear whether certain gene expression types have an influence on the outcome of CRC. It was recently shown that the gene phosphatidylinositol-4, 5-bisphosphate 3-kinase catalytic subunit alpha (PIK3CA) and its mutation could be associated with patient survival (Domingo et al. 2013). Wu et al. (Wu et al. 2013) conducted a meta-analysis and demonstrated that PIK3CA mutation is associated with poor survival for patients with metastatic CRC. If there is an association between gene expression and outcome, further research could help to find new therapies for CRC.

Aspirin's mechanism of action is based on the irreversible inactivation of cyclooxygenase, a prostaglandin oxidase reductase, with two isoenzymes: COX-1 and COX-2. Due to the suppression of prostaglandin and thromboxane, it has effects on platelet aggregation and inflammation. Reducing the prostaglandin E2 (PGE2) production of COX-2 can decrease tumour cell proliferation by different pathways. COX-2 is therefore also known as prostaglandin-endoperoxide synthase 2 or PTGS2. The inhibition of COX-1 in platelets results in a lower production of thromboxane A2 (TXA2) and vascular endothelial growth factor (VEGF), which reduces angiogenesis and metastasis of the tumour. Additionally, aspirin inhibits the nuclear translocation of nuclear factor kappa-light-chain-enhancer of activated $B$ cells (NF- $\mathrm{\kappa B}$ ) and supports apoptosis of tumour cells by that pathway. It also induces apoptosis by affecting the ratio of B-cell lymphoma protein 2-associated X (Bax) to B-cell lymphoma protein 2 (Bcl-2). Moreover, it was found that aspirin enhances the expression of death receptor 5 (DR5), which yields another way to increase apoptosis. By inhibiting the mammalian target of rapamycin (mTOR) and activating AMP-activated protein kinase (AMPK), aspirin has a positive effect on autophagy in CRC cell models. In addition, aspirin stimulates the DNA mismatch repair (MMR) system and has been reported to suppress oxidative stress (Ma et al. 2017). Overexpression of COX-2 has been found in CRC cells, which might be an important point of action (Di Francesco et al. 2015). It is still a subject of debate whether or not aspirin has the potential to reduce the incidence of CRC (Bosetti et al. 2012). Due to negative side effects such as gastrointestinal bleeding, it is not recommended as primary prevention. According to many clinical studies published in the last few years, aspirin is becoming more important as a new therapy of CRC. Today, the standard care in CRC consists of surgery, radiotherapy and chemoradiotherapy. Furthermore, fluorouracil-based adjuvant chemotherapy is recommended for patients with stage III colon cancer which has been completely resected or high-risk stage II colon cancer (Weitz et al. 2005).

One side effect of aspirin therapy is gastrointestinal bleeding (Haykal et al. 2019). We evaluated relevant bleeding events described in the studies in this review, and we highlighted the advantages and disadvantages of aspirin. Against this background, we conducted a meta-analysis to present the current state of aspirin-guided CRC therapy.

\section{Materials and methods}

\section{Literature search and study selection}

A protocol for the systematic search strategy was prepared in advance by J.C.M. and P.S. This included explanations of the study synopsis, the medical problem, design aspects, statistical analysis and information synthesis. The Preferred Reporting Items for Systematic Reviews and Meta-Analyses (PRISMA) statement was used to report the results (Moher et al. 2009). The systematic search was carried out by two independent reviewers in the databases PubMed and Web of Science. The former was conducted by J.C.M. and L.K. In case of any disagreement, the matter was discussed with P.S., and then, a consensus was found. The literature sources were managed with Endnote. We included relevant studies published before July 2019. Titles and abstracts were scanned, and where appropriate, the full papers were read and the inclusion criteria evaluated. For our analysis, we searched for randomised controlled trials, because they are the preferred design for studying effects and they are more likely to provide unbiased information than observational study designs (Reeves et al. 2020). Additionally, cohort studies and population-based case-control studies were accepted.

The following keywords were used: "Aspirin" AND "Colon cancer"; "Aspirin" AND "Colon cancer" AND "Prognosis"; "Aspirin" AND "Colon cancer" AND "Outcome"; "Aspirin" AND "Colon cancer" AND "postoperative". Those keywords were selected, because patients with $\mathrm{CRC}$ and aspirin therapy were prerequisites for inclusion and because we wanted to clarify the results with additional criteria that would yield outcome data regarding the prognoses 
of patients who mostly underwent surgery. The search strategy addressed the influence of different genes on patient survival including PIK3CA. The investigation of other genes, such as PTGS2 (COX-2), depended on the availability of data in the respective studies. Uncontrolled studies and review articles were excluded from the results. Only studies with new primary data were included. Furthermore, some results of the literature search were excluded after the full texts were read in case where no patient survival data were available. As an expert in gastroenterology, A.S. was involved in the process of finding unpublished literature. After having finished the search, we also scanned interesting reviews and studies for missed matching studies.

\section{Data extraction}

Based on a structured data extraction sheet, data were extracted and subsequently compared, and disagreements were resolved. The following items were deemed to be relevant: study ID, citation, design, duration, blinding, number and characteristics of participants, interventions, outcomes/ results, adverse outcomes, instruments/scales applied, relative risk, and odds ratios with the corresponding error or 95\% confidence intervals. The Cochrane Collaboration's tool for assessing risk of bias was used. The Newcastle Ottawa Scale was used to evaluate study quality (Stang 2010).

\section{Statistical analysis}

Published hazard ratios (HR) and odds ratios from casecontrol studies were combined using the inverse variance method. Heterogeneity was measured using Cochrane's $Q$ test, the $I^{2}$ measure, and the heterogeneity variance based on the random effects model according to DerSimonian and Laird (DerSimonian and Laird 1986). Hazard ratios were extracted with their corresponding 95\% confidence intervals. Since a meta-analysis is an observational study, the statistical analysis covered the investigation of bias, chance, and confounding. We classified each study for its level of evidence.

The results were presented graphically in a forest plot. Forest plots were presented by intervention. Other descriptive measures include confounder variables, means of statistical analysis, study design and publication year or performing year.

At the first stage, the data were visualised with a funnel plot which is a scatter plot of sample size and effect sizes. A more formal analysis of publication bias (i.e., file-drawer bias which refers to the possibility that only positive studies are published especially in the case of small studies) was based on Egger's test or other appropriate methods (Deeks et al. 2005). A summary HR was determined together with a $95 \%$ confidence interval. The cut-off for statistical significance was set at $p<0.05$. Additionally, all estimates were separated by intervention. In this meta-analysis we used the random effects model to calculate the global result of the effect measures of each study (Schwarzer 2015). If no heterogeneity was present, a fixed-effects model was applied. Subgroup analyses were added for different gene expressions in patients. Cochrane's $Q$ test was applied as an initial test for heterogeneity. Based on this test, the percentages of total variation across studies which are due to heterogeneity rather than chance $\left(\mathrm{I}^{2}\right)$ were estimated and presented. Calculations were performed with R 3.6.3 (R Core Team 2019) and the package meta (Balduzzi et al. 2019). For all studies which are not randomised controlled trials (RCTs), we investigated age, sex, stage, aspirin dosage, and different gene expressions of the given population. The analysis was redone by leaving out one study for sensitivity analysis.

\section{Results}

\section{Studies selected for the review}

In total, we selected 27 studies in our meta-analysis which fulfilled the inclusion criteria. Two studies were case-control studies and the remaining 25 were cohort studies. Due to the low number of case-control studies, we evaluated them together with the cohort studies as a single group. No completed randomised controlled study was found. For a few studies, it was difficult to obtain the full texts. The local university library was consulted for help. For some publications, especially very old ones, the full texts were located in this way. The remaining results without a successful full-text search were excluded. During the literature search, which included randomised controlled studies, we found several study protocols of ongoing studies.

The total sample size was 237,245 patients. Of the selected studies, 19 were used for the analysis of postdiagnosis aspirin use and 12 for the analysis of aspirin use before CRC diagnosis. The included studies were published between 2009 and 2017. Further information, such as study design, country, aspirin dosage, sample size and adjustments, and those regarding age, sex, cancer type, and stage can be found in Table 1 'Study characteristics' and its complete version (Supplementary, Table 2). We extracted data for overall-, recurrence-free- and CRC-specific survival of patients with CRC. Furthermore, we categorised the data into two groups: the patients with CRC who were taking aspirin before their diagnosis (e.g., for cardiovascular prevention), hereafter called 'prediagnosis Aspirin use', and the patients who used aspirin after their diagnosis of CRC ('postdiagnosis Aspirin use'). Additionally, in the postdiagnosis aspirin use group, we made a subgroup analysis of certain gene expressions. For that, 
Table 1 Study characteristics (for full data sheet, see Supplementary Table 2)

\begin{tabular}{|c|c|c|c|c|c|c|c|c|c|}
\hline Study & Year & Sample size & Gene analysis & Dose (mg) & $\begin{array}{l}\text { pre-/postdiag- } \\
\text { nosis }\end{array}$ & Cancer type & Stage & Outcome & Study quality \\
\hline $\begin{array}{l}\text { Bains et al. } \\
\text { (2016) }\end{array}$ & 2016 & 23,162 & None & $75 / 160$ & Post & $\mathrm{CRC}$ & I-IV & $\begin{array}{l}\text { All-cause } \\
\text { deaths: } 9289 \text {, } \\
\text { CRC-specific } \\
\text { deaths: } 6533\end{array}$ & 9 \\
\hline $\begin{array}{l}\text { Bastiaannet } \\
\text { et al. (2012) }\end{array}$ & 2012 & 4481 & None & $80 / 30$ & Post & $\mathrm{CRC}$ & I-IV & n.a. & 8 \\
\hline $\begin{array}{l}\text { Cardwell et al. } \\
\text { (2014) }\end{array}$ & 2014 & 12,868 & None & $\begin{array}{l}25(0.3 \%) / 75 \\
\quad(98.5 \%) />300 \\
(1.2 \%)\end{array}$ & Pre + post & $\mathrm{CRC}$ & I-IV & $\begin{array}{l}\text { All-cause } \\
\text { deaths: } 2214 \text {, } \\
\text { CRC-specific } \\
\text { deaths: } 1559\end{array}$ & 4 \\
\hline $\begin{array}{c}\text { Chan et al. } \\
\text { (2009) }\end{array}$ & 2009 & 1279 & None & 325 & Pre + post & $\mathrm{CRC}$ & I-III & $\begin{array}{l}\text { All-cause } \\
\text { deaths: } 480 \text {, } \\
\text { CRC-specific } \\
\text { deaths: } 222\end{array}$ & 7 \\
\hline $\begin{array}{l}\text { Domingo et al. } \\
\text { (2013) }\end{array}$ & 2013 & 896 & PIK3CA & $<100$ & Post & $\mathrm{CRC}$ & II-III & $\begin{array}{l}\text { All-cause } \\
\text { deaths: } 395\end{array}$ & 8 \\
\hline $\begin{array}{l}\text { Frouws et al. } \\
\text { (2017) }\end{array}$ & 2017 & 599 & BRAF, KRAS & $80-100$ & Post & $\mathrm{CC}$ & I-IV & $\begin{array}{l}\text { All-cause } \\
\text { deaths: } 267\end{array}$ & 8 \\
\hline $\begin{array}{l}\text { Goh et al. } \\
\text { (2014) }\end{array}$ & 2014 & 726 & None & 100 & Pre + post & CRC & I-III & $\begin{array}{r}\text { CRC-specific } \\
\text { deaths: } 181\end{array}$ & 8 \\
\hline $\begin{array}{c}\text { Gray et al. } \\
\text { (2017) }\end{array}$ & 2017 & 680 & $\begin{array}{l}\text { PTGS2, } \\
\text { PIK3CA }\end{array}$ & 75 & Post & $\mathrm{CRC}$ & II-III & $\begin{array}{l}\text { All-cause } \\
\text { deaths: } 299 \text {, } \\
\text { CRC-specific } \\
\text { deaths: } 212\end{array}$ & 8 \\
\hline $\begin{array}{l}\text { Hamada et al. } \\
\text { (2017) }\end{array}$ & 2017 & 617 & CD274 & $81 / 325$ & Post & $\mathrm{CRC}$ & I-IV & $\begin{array}{l}\text { All-cause } \\
\text { deaths: } 325 \text {, } \\
\text { CRC-specific } \\
\text { deaths: } 118\end{array}$ & 6 \\
\hline $\begin{array}{l}\text { Hua et al. } \\
\text { (2017) }\end{array}$ & 2017 & 2419 & KRAS, BRAF & n.a. & Pre + post & $\mathrm{CRC}$ & I-IV & $\begin{array}{l}\text { All-cause } \\
\text { deaths: } 381 \text {, } \\
\text { CRC-specific } \\
\text { deaths: } 100\end{array}$ & 8 \\
\hline $\begin{array}{l}\text { Liao et al. } \\
\quad(2012)\end{array}$ & 2012 & 964 & $\begin{array}{l}\text { PIK3CA, } \\
\text { KRAS, } \\
\text { BRAF, } \\
\text { PTGS2, } \\
\text { CIMP, LINE- } \\
\text { 1, phospho- } \\
\text { rylated AKT }\end{array}$ & 325 & Post & $\mathrm{CRC}$ & I-IV & $\begin{array}{l}\text { All-cause } \\
\text { deaths: } 395 \text {, } \\
\text { CRC-specific } \\
\text { deaths: } 190\end{array}$ & 6 \\
\hline $\begin{array}{l}\text { McCowan et al. } \\
\text { (2013) }\end{array}$ & 2013 & 2990 & None & $75 / 300$ & Pre + post & $\mathrm{CRC}$ & I-IV & $\begin{array}{l}\text { All-cause } \\
\text { deaths: } 1998 \text {, } \\
\text { CRC-specific } \\
\text { deaths: } 1021\end{array}$ & 9 \\
\hline Ng et al. (2015) & 2015 & 799 & None & n.a. & Post & $\mathrm{CC}$ & III & $\begin{array}{l}\text { All-cause } \\
\text { deaths: } 156\end{array}$ & 6 \\
\hline $\begin{array}{l}\text { Reimers et al. } \\
\text { (2014) }\end{array}$ & 2014 & 999 & $\begin{array}{l}\text { PTGS2, } \\
\text { PIK3CA, } \\
\text { HLA class I }\end{array}$ & $75-325$ & Post & $\mathrm{CC}$ & I-IV & $\begin{array}{l}\text { All-cause } \\
\text { deaths: } 465\end{array}$ & 8 \\
\hline $\begin{array}{l}\text { Walker et al. } \\
\text { (2012) }\end{array}$ & 2012 & 13,944 & None & $75 />75$ & Pre + post & CRC & I-IV & $\begin{array}{l}\text { All-cause } \\
\text { deaths: } 5358\end{array}$ & 8 \\
\hline $\begin{array}{l}\text { Coghill, et al. } \\
(2011 \mathrm{a}, \mathrm{b})\end{array}$ & 2011 & 1737 & None & n.a. & Pre & $\mathrm{CRC}$ & I-IV & $\begin{array}{l}\text { All-cause } \\
\text { deaths: } 707 \text {, } \\
\text { CRC-specific } \\
\text { deaths: } 262\end{array}$ & 8 \\
\hline
\end{tabular}


Table 1 (continued)

\begin{tabular}{|c|c|c|c|c|c|c|c|c|c|}
\hline Study & Year & Sample size & Gene analysis & Dose (mg) & $\begin{array}{l}\text { pre-/postdiag- } \\
\text { nosis }\end{array}$ & Cancer type & Stage & Outcome & Study quality \\
\hline $\begin{array}{c}\text { Coghill, et al. } \\
(2011 \mathrm{a}, \mathrm{b})\end{array}$ & 2011 & 1051 & None & n.a. & Pre & CRC & I-IV & $\begin{array}{l}\text { All-cause } \\
\text { deaths: } 371 \text {, } \\
\text { CRC-specific } \\
\text { deaths: } 274\end{array}$ & 9 \\
\hline $\begin{array}{l}\text { Coghill et al. } \\
\text { (2012) }\end{array}$ & 2012 & 160,143 & None & $<200->325 \mathrm{mg}$ & Pre & CRC & I-IV & $\begin{array}{l}\text { All-cause } \\
\text { deaths: } \\
\text { 15,608, } \\
\text { CRC-specific } \\
\text { deaths: } 492\end{array}$ & 7 \\
\hline $\begin{array}{l}\text { Din et al. } \\
(2010)\end{array}$ & 2010 & 2259 & None & 75 & Pre & CRC & I-IV & $\begin{array}{l}\text { All-cause } \\
\text { deaths: } 670 \text {, } \\
\text { CRC-specific } \\
\text { deaths: } 561\end{array}$ & 5 \\
\hline $\begin{array}{l}\text { Giampieri et al. } \\
\text { (2017) }\end{array}$ & 2017 & 66 & KRAS, BRAF & 100 & Pre & CRC & I-III & $\begin{array}{l}\text { All-cause } \\
\text { deaths: } 66\end{array}$ & 8 \\
\hline $\begin{array}{l}\text { Hippisley-Cox } \\
\text { and Coupland } \\
\text { (2017) }\end{array}$ & 2017 & 44,145 & None & n.a. & Pre & $\mathrm{CRC}$ & I-IV & $\begin{array}{l}\text { All-cause } \\
\text { deaths: } \\
26,887 \text {, } \\
\text { CRC-specific } \\
\text { deaths: } \\
\text { 13,588 } \\
\text { (derivation } \\
\text { cohort) }\end{array}$ & 8 \\
\hline $\begin{array}{l}\text { Kim et al. } \\
\text { (2015) }\end{array}$ & 2015 & 686 & None & n.a. & Pre & CRC & III & n.a. & 8 \\
\hline $\begin{array}{l}\text { Kothari et al. } \\
\text { (2015) }\end{array}$ & 2015 & 1487 & PIK3CA & $81-325$ & Pre & CRC & I-IV & n.a. & 8 \\
\hline $\begin{array}{l}\text { Murphy et al. } \\
\text { (2017) }\end{array}$ & 2017 & 488 & PIK3CA & $>75$ & Post & $\mathrm{CC}$ & II & $\begin{array}{l}\text { All-cause } \\
\text { deaths } \\
\text { (PIK3CA- } \\
\text { Mutation): } \\
\text { 17; all-cause } \\
\text { deaths } \\
\text { (PIK3CA- } \\
\text { Wildtype.): } \\
80\end{array}$ & 8 \\
\hline $\begin{array}{l}\text { Zell et al. } \\
\text { (2009) }\end{array}$ & 2009 & 621 & None & n.a. & Pre & CRC & I-IV & $\begin{array}{l}\text { all-cause } \\
\text { deaths: } 222 \text {, } \\
\text { CRC-specific } \\
\text { deaths: } 145\end{array}$ & 7 \\
\hline $\begin{array}{l}\text { Zanders et al. } \\
\text { (2015) }\end{array}$ & 2015 & 1043 & None & $<100$ & Post & CRC & I-IV & $\begin{array}{l}\text { All-cause } \\
\text { deaths: } 494\end{array}$ & 9 \\
\hline $\begin{array}{l}\text { Restivo et al. } \\
\text { (2015) }\end{array}$ & 2015 & 241 & None & 100 & Post & $\mathrm{RC}$ & II-III & n.a. & 8 \\
\hline
\end{tabular}

we had a look at PIK3CA and PTGS2. We did not find a publication bias in our study with the use of the command 'metabias' in R. Furthermore, all subgroup analyses were repeated by leaving out one study. For that, we used the function 'metainf' in R. We did not find any study which stood out. Study quality was assessed using the Newcastle Ottawa Scale (Stang 2010). This scale tries to address the quality of studies which are not randomised and controlled by the use of three categories. These are 'selection', 'comparability' and 'outcome' for cohort studies or 'selection', 'comparability' and 'exposure' for case-control studies. The maximum number of points on the scale is 9 . The results are presented in Table 1. In conclusion, most of the studies were graded as high quality and the scores ranged between 6 and 9. They did not have relevant shortcomings. Both of the case-control studies (Cardwell et al. 2014; Din et al. 2010) had lower scores of 4 and 5 points. 


\section{Postdiagnosis aspirin use}

In total, 18 studies were used in the analysis of postdiagnosis aspirin use (including PIK3CA and PTGS2 analysis) (Fig. 1). Of those, 8 were included for CRC-specific survival, 3 for recurrence-free survival and 16 for overall survival calculations. We found an improvement of CRCspecific survival (Fig. 2) with a hazard ratio (HR) for cancer-related death of 0.74 (95\% CI: 0.62-0.89) and a substantial heterogeneity $\left(I^{2}=72 \%, \operatorname{tau}^{2}=0.0425, Q=24.89\right.$, $p<0.01)$. We did not find publication bias $(t=-2.21$, $p=0.07$ ). Our analysis of overall survival (Fig. 3 ) in the postdiagnosis aspirin use group revealed a lower mortality rate, based on an HR of 0.82 (95\% CI: 0.74-0.90). The heterogeneity amounted to $I^{2}=69 \%\left(\operatorname{tau}^{2}=0.0191, Q=48.66\right.$, $p<0.01)$. However, publication bias cannot be ruled out $(t=-2.78, p=0.015)$. We then analysed recurrence-free survival data (see Supplementary Fig. 8). Here, we found reduced mortality with an HR of 0.50 (95\% CI: 0.33-0.76) with a heterogeneity of $I^{2}=0 \%\left(\operatorname{tau}^{2}=0, Q=0.8, p=0.67\right)$. Therefore, we used the fixed-effects model. No publication bias was found $(t=-0.34, p=0.79)$.

\section{Prediagnosis aspirin use}

The analysis of prediagnosis aspirin use was based on 14 studies, 12 of which were used for CRC-specific survival and nine of which for overall survival. We should print out that for both subgroups, Hippisley-Cox et al. (HippisleyCox and Coupland 2017) distinguished between men and women. Those were assessed as two separate studies. An HR of 0.91 (95\% CI: 0.82-1.01) showed an improved overall survival and CRC-specific survival (see Supplementary Figs. 9 and 10). Heterogeneity for CRC-specific survival was calculated as $I^{2}=71 \%\left(\operatorname{tau}^{2}=0.0203, Q=40.99\right)$ with a statistical significance of $p<0.01$. There was no evidence of publication bias $(t=1.17, p=0.27)$. For overall survival, we found a considerable heterogeneity of $I^{2}=77 \%$ $\left(\operatorname{tau}^{2}=0.0147, Q=39.51, \mathrm{p}<0.01\right)$; no publication bias was detected $(t=-0.31, p=0.77)$.

\section{PIK3CA}

For the analysis of postdiagnosis aspirin use regarding the PIK3CA mutation status, we found 5 corresponding studies. The pooled HR for cancer-related death
Fig. 1 PRISMA flow diagram (Moher et al. 2009)

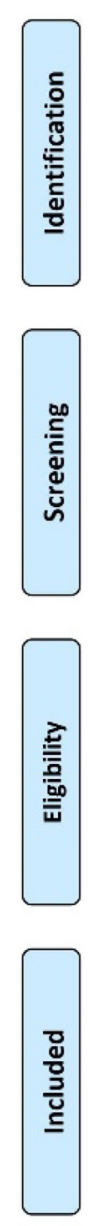

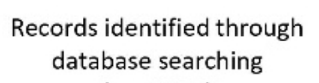

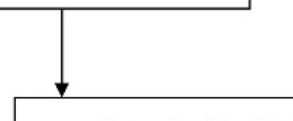

Records after duplicates removed $(n=2215)$

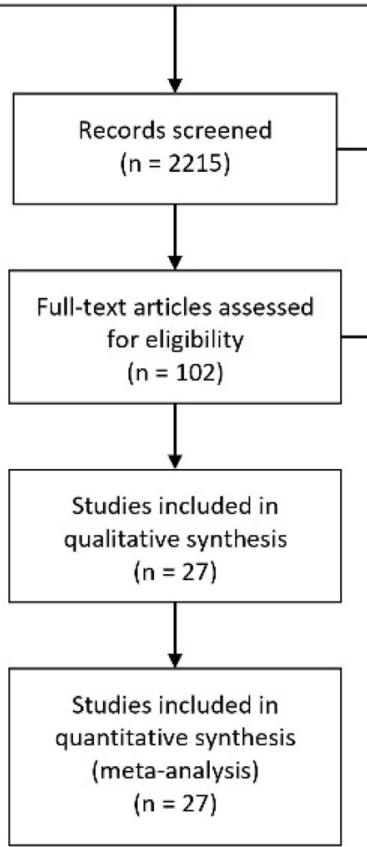

Additional records identified through other sources $(n=0)$

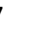


considering PIK3CA-mutated tumours (Fig. 4) was calculated as 0.74 (95\% CI: 0.56-0.97) with a heterogeneity of $I^{2}=0 \%\left(\operatorname{tau}^{2}=0, Q=3.99, p=0.41\right)$. For that reason, we selected the fixed-effects model. Publication bias was not found $(t=-0.15, p=0.89)$. PIK3CA wild-type status
(Fig. 5) was associated with an HR of 0.98 (95\% CI: $0.62-1.53)$ and a considerable heterogeneity of $I^{2}=82 \%$ $\left(\operatorname{tau}^{2}=0.2013, Q=22.6\right)$ and statistical significance of $p<0.01$. We did not find publication bias $(t=0.51$, $p=0.64)$.
Fig. 2 Postdiagnosis aspirin use, overall survival
Fig. 3 Postdiagnosis aspirin use, CRC-specific survival

Fig. 4 Postdiagnosis aspirin use, PIK3CA mutation
Study

Reimers et al. 2014

Hamada et al. 2017

Hua et al. 2017

Gray et al. 2017

Frouws et al. 2017

Bains et al. 2016

$\mathrm{Ng}$ et al. 2015

Cardwell et al. 2014

McCowan et al. 2013

Walker et al. 2012

Bastiaannet et al. 2012

Zanders et al. 2015

Domingo et al. 2013

Restivo et al. 2015

Murphy et al. 2017

Random effects model
Chan et al. 2009 $\log \mathrm{HR}$ se(logHR)

-0.45
-0.24
-0.30
-0.30
-0.27
-0.45
-0.05
-0.46
0.06
-0.40
-0.09
-0.26
-0.04
-0.13
-1.56
0.23

0.1372

0.1043

0.2029

0.1213

0.1541

0.1519

0.0300

0.3029

0.0614

0.0850

0.0517

0.1070

0.1421

0.2657

0.7498

0.2921

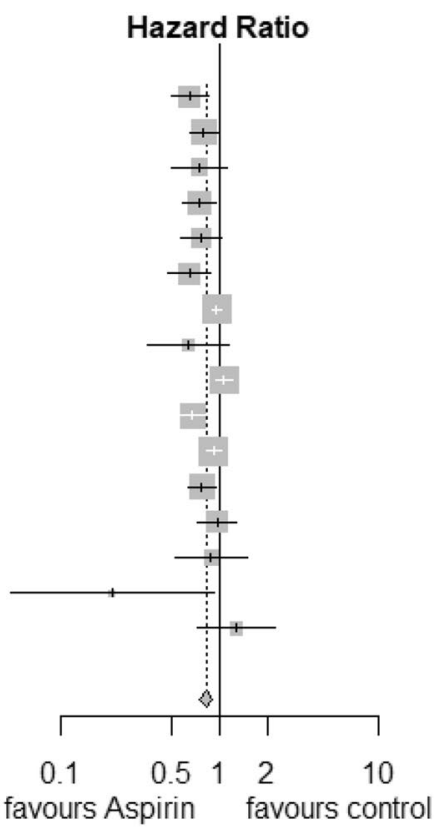

HR $\quad 95 \%-\mathrm{Cl}$

$0.64 \quad[0.49 ; 0.84]$

$0.79[0.64 ; 0.97]$

$0.74[0.50 ; 1.10]$

$0.74 \quad[0.58 ; 0.94]$

$0.76 \quad[0.56 ; 1.03]$

$0.64 \quad[0.48 ; 0.86]$

$0.95 \quad[0.90 ; 1.01]$

$0.63[0.35 ; 1.14]$

$1.06[0.94 ; 1.20]$

$0.67[0.57 ; 0.79]$

$0.91 \quad[0.82 ; 1.01]$

$0.77[0.62 ; 0.95]$

$0.96[0.73 ; 1.27]$

$0.88 \quad[0.52 ; 1.48]$

$0.21 \quad[0.05 ; 0.91]$

$1.26[0.71 ; 2.23]$

$0.82[0.74 ; 0.90]$

favours Aspirin favours control
Study

Chan et al. 2009

Hamada et al. 2017

Hua et al. 2017

Gray et al. 2017

Bains et al. 2016

Goh et al. 2014

Cardwell et al. 2014

McCowan et al. 2013

Random effects model $\log \mathrm{HR}$ se(logHR)

$-0.34$

$-0.43$

$-0.82$

$-0.37$

$-0.16$

$-0.21$

0.06

$-0.54$
0.1520

0.2562

0.2930

0.1914

0.0638

0.2396

0.0777

0.1330

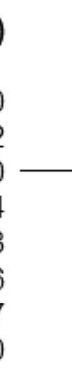

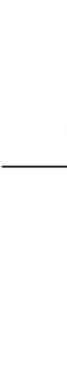

\section{Hazard Ratio}

HR

$95 \%-\mathrm{Cl}$

$0.71 \quad[0.53 ; 0.96]$

$0.65 \quad[0.39 ; 1.07]$

$0.44[0.25 ; 0.78]$

$0.69 \quad[0.47 ; 1.00]$

$0.85[0.75 ; 0.96]$

$0.81[0.51 ; 1.30]$

$1.06[0.91 ; 1.23]$

$0.58[0.45 ; 0.75]$

$0.74[0.62 ; 0.89]$ favours Aspirin favours control

Study

Reimers et al. 2014

Gray et al. 2017

Liao et al. 2012

Domingo et al. 2013

Murphy 2017

Fixed effect model $\log \mathrm{HR}$ se(logHR)

$-0.31 \quad 0.4159$

$-0.22$

$-0.62$

$-1.24$

0.57

0.1782

0.2889

1.0471

0.6437

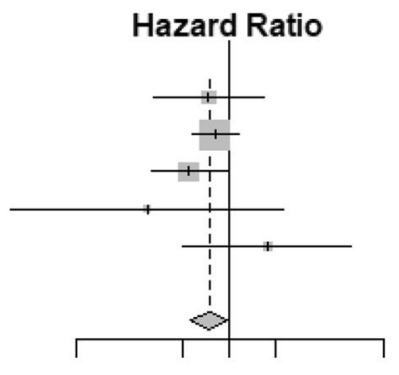

$\begin{array}{lllll}0.1 & 0.5 & 1 & 2 & 10\end{array}$

favours Aspirin favours control $\begin{array}{rr}\text { HR } & \mathbf{9 5 \%} \mathbf{r}-\mathbf{C l} \\ & \\ 0.73 & {[0.32 ; 1.65]} \\ 0.80 & {[0.56 ; 1.13]} \\ 0.54 & {[0.31 ; 0.95]} \\ 0.29 & {[0.04 ; 2.26]} \\ 1.76 & {[0.50 ; 6.21]}\end{array}$

$0.74[0.56 ; 0.97]$ 


\section{PTGS2}

Only 3 studies were recruited for further analysis of PTGS2 (COX-2) status in the postdiagnosis aspirin use group. For high expression of PTGS2 (Fig. 6) we calculated a reduction in mortality with a pooled HR of 0.65 (95\% CI: $0.52-0.82$ ) with a heterogeneity of $I^{2}=0 \%\left(\operatorname{tau}^{2}=0, Q=0.12, p=0.94\right)$. Here, we also used the fixed-effects model, since no heterogeneity was found. No publication bias was assessed $(t=-1.31, p=0.41)$. Low expression of PTGS2 (Fig. 7) was associated with an HR of 0.91 (95\% CI: 0.55-1.51) and a heterogeneity of $I^{2}=65 \%\left(\operatorname{tau}^{2}=0.1309, Q=5.75, p=0.06\right)$. Likewise, no publication bias was found $(t=0.42, p=0.74)$.

\section{Side effects of aspirin use}

We also examined the relevant side effects, especially bleeding, which were described in the selected studies. In total, only a few publications commented on this issue. Frouws et al. (Frouws et al. 2017) demonstrated that low-dose aspirin therapy for cardiovascular prevention doubled the incidence of gastric bleeding. In their study, they reported that 1 or 2 per thousand individuals are likely to have a gastric bleeding event per year and even up to 7 per thousand for patients older than 80 years. Giampieri et al. (Giampieri et al. 2017) did not observe intestinal bleeding in either the group of patients receiving aspirin or in the control group.

\section{Discussion}

The results of our meta-analysis indicate that aspirin use after a diagnosis of CRC was associated with better overall survival and in contrast to the meta-analysis of $\mathrm{Li}$ et al. (Li et al. 2015) even better CRC-specific survival than standard therapy. For recurrence-free survival, aspirin use after diagnosis seems to have a positive effect on patient's outcome. However, we must remark that only three studies presented usable data. The analysis of overall survival regarding PIK3CA gene expression favours
Fig. 5 Postdiagnosis aspirin use, PIK3CA wild-type

\begin{tabular}{lrr} 
Study & \multicolumn{2}{c}{ logHR se(logHR) } \\
Reimers et al. 2014 & -0.60 & 0.1637 \\
Gray et al. 2017 & -0.24 & 0.4236 \\
Liao et al. 2012 & -0.06 & 0.1158 \\
Domingo et al. 2013 & -0.05 & 0.2750 \\
Murphy 2017 & 0.92 & 0.2801
\end{tabular}

Random effects model

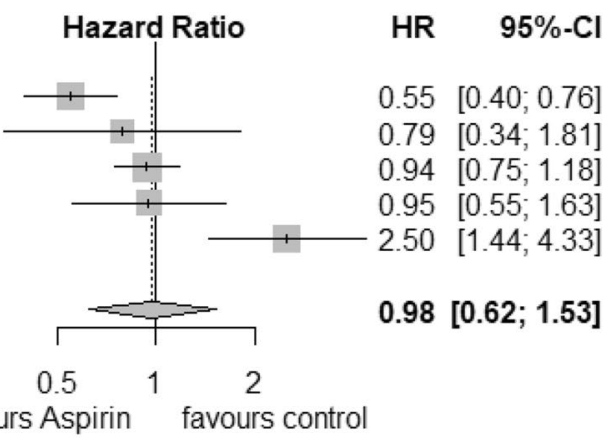

favours Aspirin favours control
Fig. 6 Postdiagnosis aspirin use, high PTGS2
Fig. 7 Postdiagnosis aspirin use, low PTGS2
Study

Reimers et al. $2014 \quad-0.39$

Gray et al. $2017 \quad-0.45$

Chan et al. $2009 \quad-0.48$

Fixed effect model $\log \mathrm{HR}$ se(log $\mathrm{HR})$

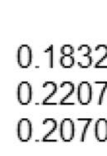

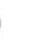

Hazard Ratio

0.2207

0.2070

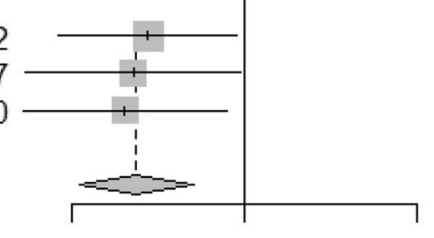

$\begin{array}{lcc}0.5 & 1 & 2 \\ \text { vours Aspirin } & \text { favours control }\end{array}$
HR $\quad 95 \%-\mathrm{Cl}$

$0.68 \quad[0.47 ; 0.97]$

$0.64[0.42 ; 0.99]$

$0.62[0.41 ; 0.93]$

$0.65[0.52 ; 0.82]$
Study

Reimers et al. 2014

Gray et al. 2017

Chan et al. 2009

Random effects model $\log \mathrm{HR}$ se(logHR)

$-0.53$

0.25

0.05

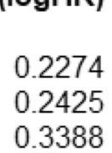

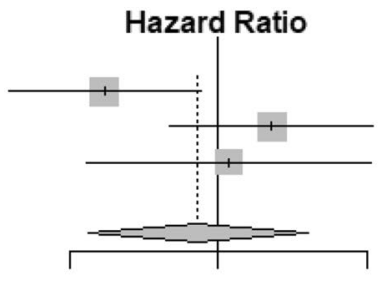

$\begin{array}{lll}0.5 & 1\end{array}$ favours Aspirin favours control
HR $\quad 95 \%-\mathrm{Cl}$

$0.59 \quad[0.38 ; 0.92]$

$1.28[0.80 ; 2.06]$

$1.05[0.54 ; 2.04]$

$0.91[0.55 ; 1.51]$ 
mutated tumours. In contrast, patients with wild-type PIK3CA status did not profit from postdiagnosis aspirin use. Additionally, PTGS2 (COX-2) status seems to be another important factor of the patient's outcome. High expression of PTGS2 is associated with better overall survival than lower expression. For prediagnosis aspirin use, we calculated the same HR for both CRC-specific survival and overall survival. We did not find a survival benefit of patients with CRC.

Until now, most studies have considered the effect of aspirin on the outcome of patients with CRC as one large group. One strength of our meta-analysis is the analysis of different genotypes. They are of special interest, since it is important to know for which group of patients an aspirin-guided therapy of CRC should be considered. Few articles studied different genes in more detail. Looking at our study, it appears that for some groups of patients, the benefit of therapy with aspirin outweighs the risk, whereas others would only be at risk of gastrointestinal bleeding. Thus, it appears that a closer look at gene expression requires further research to personalise therapy for each patient.

Furthermore, we used the Newcastle Ottawa Scale (Stang 2010) to assess study quality. It classifies the selected studies based on objective criteria, though a subjective influence remains. Most of the studies-apart from the two case-control studies-were of high quality, which is another advantage of this meta-analysis.

Due to the small number of studies, for each subgroup analysis, we presented estimated $I^{2}$, tau ${ }^{2}$ and $Q$ values to increase the transparency of our calculations. No publication bias was found in this meta-analysis. Our study was influenced by high heterogeneity of the selected studies. In contrast, PIK3CA mutation status, high expression of PTGS2 and recurrence-free survival were not biased by heterogeneity. For those three studies, the estimated $I^{2}$ was $0 \%$, so we presented the corresponding forest plots based on the fixedeffects model. This supports the thesis that patients in those groups might benefit from therapy with aspirin in addition to standard care for CRC.

We must admit that we did not find all data of interest in each study. For example, aspirin dosage is of prime importance for potential adjuvant therapy, but not all the included studies described appropriate details on medication. In most cases, there was no information given about how many patients took aspirin. The aspirin dosages varied widely, between 75 and $325 \mathrm{mg}$. However, it may be of importance for CRC patients' survival whether they have low-dose $(<300 \mathrm{mg})$ or high-dose aspirin therapy. Furthermore, higher dosages could be associated with more gastrointestinal bleeding events. In our research, we found very little information about side effect. More randomised studies are needed to find the optimal dosage for an adjuvant use and to learn more about potential side effects.
Another important factor in CRC patient outcomes is the cancer stage. Most of the selected studies used patients with stages I-IV. Only a few limited their inclusion criteria for certain groups of stage. For our analysis, we used multivariable results which were adjusted for stage in 20 of the 27 studies. Nevertheless, survival depends on the stage of CRC and it is obvious that a lower stage may be associated with better survival. In conclusion, further research on aspirin use in patients with different stages of severity is needed.

In our meta-analysis, we investigated PIK3CA and PTGS2 (COX-2). For both subgroup analyses, we must point out that the number of eligible studies was relatively small. Unfortunately, we did not find enough studies to analyse other genes such as Kirsten rat sarcoma viral oncogene (KRAS) or v-raf murine sarcoma viral oncogene homolog $\mathrm{B}$ (BRAF) which are of interest to research on CRC. Frouws et al. (Frouws et al. 2017) described a benefit for overall patient survival with wild-type-BRAF tumours $(R R=0.60$ (95\% CI: 0.44-0.83) and no association between KRAS mutation status and aspirin use after diagnosis of CRC.

Our review almost exclusively involved cohort (and two case-control studies), which provide a lower level of evidence. Cohort studies are more credible than case-control studies. Nevertheless, a few randomised controlled trials have been published regarding the concern of postdiagnosis aspirin use as adjuvant therapy for CRC. Fortunately, several studies are in process and will present further knowledge on the well-known drug acetylsalicylic acid.

We would like to emphasise that so far aspirin has only been considered as primary prevention in most studies. This has often been critically debated. In our work, we show that even after a confirmed diagnosis of CRC, there is still a therapeutic benefit with aspirin. Accordingly, this approach of tertiary prevention should not be underestimated.

\section{Conclusion}

In summary we consistently found that aspirin appears to have a favourable effect on the outcome of patients with colorectal cancer. Thus, it could be considered as a potential therapeutic approach in these patients.

Supplementary Information The online version contains supplementary material available at https://doi.org/10.1007/s00432-022-03942-1.

Author contributions Conceptualization, JCM and PS: methodology, JCM and PS; validation, JCM and PS: formal analysis, JCM and PS: investigation, JCM, L.K and PS: resources, JCM, LK and PS: data curation, JCM and PS: writing-original draft preparation, JCM, AS and PS: writing-review and editing, JCM, AS, LK and PS: visualisation, JCM and PS: supervision, JCM, AS and PS: project administration, JCM and PS. 
Funding Open Access funding enabled and organized by Projekt DEAL. This research received no external funding.

Availability of data and materials The datasets generated during or analysed during the current study are available from the corresponding author on reasonable request.

Code availability Not applicable.

\section{Declarations}

Conflicts of interest The authors declare no conflict of interest.

Open Access This article is licensed under a Creative Commons Attribution 4.0 International License, which permits use, sharing, adaptation, distribution and reproduction in any medium or format, as long as you give appropriate credit to the original author(s) and the source, provide a link to the Creative Commons licence, and indicate if changes were made. The images or other third party material in this article are included in the article's Creative Commons licence, unless indicated otherwise in a credit line to the material. If material is not included in the article's Creative Commons licence and your intended use is not permitted by statutory regulation or exceeds the permitted use, you will need to obtain permission directly from the copyright holder. To view a copy of this licence, visit http://creativecommons.org/licenses/by/4.0/.

\section{References}

Bains SJ, Mahic M, Myklebust TA, Smastuen MC, Yaqub S, Dorum LM, Bjornbeth BA, Moller B, Brudvik KW, Tasken K (2016) Aspirin as secondary prevention in patients with colorectal cancer: an unselected population-based study. J Clin Oncol 34:2501

Balduzzi S, Rücker G, Schwarzer G (2019) How to perform a metaanalysis with R: a practical tutorial. Evid Based Ment Health 22:153-160

Bastiaannet E, Sampieri K, Dekkers OM, de Craen AJ, van Herk-Sukel MP, Lemmens V, van den Broek CB, Coebergh JW, Herings RM, van de Velde CJ, Fodde R, Liefers GJ (2012) Use of aspirin postdiagnosis improves survival for colon cancer patients. $\mathrm{Br} \mathrm{J}$ Cancer 106:1564-1570

Bosetti C, Rosato V, Gallus S, Cuzick J, La Vecchia C (2012) Aspirin and cancer risk: a quantitative review to 2011. Ann Oncol 23:1403-1415

Cardwell CR, Kunzmann AT, Cantwell MM, Hughes C, Baron JA, Powe DG, Murray LJ (2014) Low-dose aspirin use after diagnosis of colorectal cancer does not increase survival: a case-control analysis of a population-based cohort. Gastroenterology 146:700

Chan AT, Ogino S, Fuchs CS (2009) Aspirin use and survival after diagnosis of colorectal cancer. Jama-J Am Med Assoc 302:649-659

Coghill AE, Newcomb PA, Campbell PT, Burnett-Hartman AN, Adams SV, Poole EM, Potter JD, Ulrich CM (2011a) Prediagnostic nonsteroidal anti-inflammatory drug use and survival after diagnosis of colorectal cancer. Gut 60:491-498

Coghill AE, Newcomb PA, Chia VM, Zheng Y, Wernli KJ, Passarelli MN, Potter JD (2011b) Pre-diagnostic NSAID use but not hormone therapy is associated with improved colorectal cancer survival in women. Br J Cancer 104:763-768

Coghill AE, Phipps AI, Bavry AA, Wactawski-Wende J, Lane DS, LaCroix A, Newcomb PA (2012) The association between NSAID use and colorectal cancer mortality: results from the women's health initiative. Cancer Epidemiol Biomark Prev 21:1966-1973

Deeks JJ, Macaskill P, Irwig L (2005) The performance of tests of publication bias and other sample size effects in systematic reviews of diagnostic test accuracy was assessed. J Clin Epidemiol 58:882-893

DerSimonian R, Laird N (1986) Meta-analysis in clinical trials. Control Clin Trials 7:177-188

Di Francesco L, Contreras LAL, Sacco A, Patrignani P (2015) New insights into the mechanism of action of aspirin in the prevention of colorectal neoplasia. Curr Pharm Des 21:5116-5126

Din FV, Theodoratou E, Farrington SM, Tenesa A, Barnetson RA, Cetnarskyj R, Stark L, Porteous ME, Campbell H, Dunlop MG (2010) Effect of aspirin and NSAIDs on risk and survival from colorectal cancer. Gut 59:1670-1679

Domingo E, Church DN, Sieber O, Ramamoorthy R, Yanagisawa Y, Johnstone E, Davidson B, Kerr DJ, Tomlinson IP, Midgley R (2013) Evaluation of PIK3CA mutation as a predictor of benefit from nonsteroidal anti-inflammatory drug therapy in colorectal cancer. J Clin Oncol 31:4297-4305

Frouws MA, Reimers MS, Swets M, Bastiaannet E, Prinse B, van Eijk R, Lemmens VEPP, van Herk-Sukel MPP, van Wezel T, Kuppen PJK, Morreau H, van de Velde CJH, Liefers GJ (2017) The influence of BRAF and KRAS mutation status on the association between aspirin use and survival after colon cancer diagnosis. PLoS ONE 12:e0170775

Giampieri R, Restivo A, Pusceddu V, Del Prete M, Maccaroni E, Bittoni A, Faloppi L, Andrikou K, Bianconi M, Cabras F, Berardi R, Zorcolo L, Scintu F, Cascinu S, Scartozzi M (2017) The role of aspirin as antitumoral agent for heavily pretreated patients with metastatic colorectal cancer receiving capecitabine monotherapy. Clin Colorectal Cancer 16:38-43

Goh CH, Leong WQ, Chew MH, Pan YS, Tony LK, Chew L, Tan IB, Toh HC, Tang CL, Fu WP, Chia WK (2014) Post-operative aspirin use and colorectal cancer-specific survival in patients with stage I-III colorectal cancer. Anticancer Res 34:7407-7414

Gray RT, Cantwell MM, Coleman HG, Loughrey MB, Bankhead P, McQuaid S, O'Neill RF, Arthur K, Bingham V, McGready C, Gavin AT, Cardwell CR, Johnston BT, James JA, Hamilton PW, Salto-Tellez M, Murray LJ (2017) Evaluation of PTGS2 expression, PIK3CA mutation, aspirin use and colon cancer survival in a population-based cohort study. Clin Transl Gastroenterol 8:e91

Hamada T, Cao Y, Qian ZR, Masugi Y, Nowak JA, Yang J, Song M, Mima K, Kosumi K, Liu L, Shi Y, da Silva A, Gu M, Li W, Keum N, Zhang X, Wu K, Meyerhardt JA, Giovannucci EL, Giannakis M, Rodig SJ, Freeman GJ, Nevo D, Wang M, Chan AT, Fuchs CS, Nishihara R, Ogino S (2017) Aspirin use and colorectal cancer survival according to Tumor CD274 (programmed cell death 1 ligand 1) expression status. J Clin Oncol 35:1836-1844

Haykal T, Barbarawi M, Zayed Y, Yelangi A, Dhillon H, Goranta S, Kheiri B, Chahine A, Samji V, Kerbage J, Katato K, Bachuwa G (2019) Safety and efficacy of aspirin for primary prevention of cancer: a meta-analysis of randomized controlled trials. J Cancer Res Clin Oncol 145:1795-1809

Hippisley-Cox J, Coupland C (2017) Development and validation of risk prediction equations to estimate survival in patients with colorectal cancer: cohort study. BMJ 357:j2497. https://doi.org/ 10.1136/bmj.j2497

Hua XW, Phipps AI, Burnett-Hartman AN, Adams SV, Hardikar S, Cohen SA, Kocarnik JM, Ahnen DJ, Lindor NM, Baron JA, Newcomb PA (2017) Timing of aspirin and other nonsteroidal antiinflammatory drug use among patients with colorectal cancer in relation to tumor markers and survival. J Clin Oncol 35:2806 
Kim B, Park SJ, Hong SP, Cheon JH, Kim WH, Kim TI (2015) The effect of prediagnostic aspirin use on the prognosis of stage III colorectal cancer. Int J Clin Exp Med 8:13435-13445

Kothari N, Kim R, Jorissen RN, Desai J, Tie J, Wong H-L, Farragher I, Jones I, Day FL, Li S, Sakthinandeswaren A, Palmieri M, Lipton L, Schell M, Teer JK, Shibata D, Yeatman T, Sieber OM, Gibbs P, Tran B (2015) Impact of regular aspirin use on overall and cancerspecific survival in patients with colorectal cancer harboring a PIK3CA mutation. Acta Oncol 54:487-492

Li P, Wu H, Zhang H, Shi Y, Xu J, Ye Y, Xia D, Yang J, Cai J, Wu Y (2015) Aspirin use after diagnosis but not prediagnosis improves established colorectal cancer survival: a meta-analysis. Gut 64:1419-1425

Liao X, Lochhead P, Nishihara R, Morikawa T, Kuchiba A, Yamauchi M, Imamura Y, Qian ZR, Baba Y, Shima K, Sun R, Nosho K, Meyerhardt JA, Giovannucci E, Fuchs CS, Chan AT, Ogino S (2012) Aspirin use, tumor PIK3CA mutation, and colorectalcancer survival. N Engl J Med 367:1596-1606

Ma J, Cai Z, Wei H, Liu X, Zhao Q, Zhang T (2017) The anti-tumor effect of aspirin: what we know and what we expect. Biomed Pharmacother 95:656-661

McCowan C, Munro AJ, Donnan PT, Steele RJC (2013) Use of aspirin post-diagnosis in a cohort of patients with colorectal cancer and its association with all-cause and colorectal cancer specific mortality. Eur J Cancer 49:1049-1057

Michel P, Boige V, Andre T, Aparicio T, Bachet JB, Dahan L, Guimbaud R, Lepage C, Manfredi S, Tougeron D, Taieb J, Selves J, Le Malicot K, Di Fiore F, Maillard E (2018) Aspirin versus placebo in stage III or high-risk stage II colon cancer with PIK3CA mutation: a French randomised double-blind phase III trial (PRODIGE 50-ASPIK). Dig Liver Dis 50:305-307

Moher D, Liberati A, Tetzlaff J, Altman DG (2009) Prisma group "preferred reporting items for systematic reviews and meta-analyses: the PRISMA statement." PLoS Med 6:e1000097

Murphy C, Turner N, Wong HL, Sinnathamby M, Tie J, Lee B, Desai J, Skinner I, Christie M, Hutchinson R, Lunke S, Waring P, Gibbs P, Tran B (2017) Examining the impact of regular aspirin use and PIK3CA mutations on survival in stage 2 colon cancer. Intern Med J 47:88-98

Ng K, Meyerhardt JA, Chan AT, Sato K, Chan JA, Niedzwiecki D, Saltz LB, Mayer RJ, Benson A, Schaefer PL, Whittom R, Hantel A, Goldberg RM, Venook AP, Ogino S, Giovannucci EL, Fuchs CS (2015) Aspirin and COX-2 inhibitor use in patients with stage III colon cancer. J Natl Cancer Inst 107:345

Reeves BC, Deeks JJ, Higgins JPT, Shea B, Tugwell P, and Wells GA (2020) Chapter 24: including non-randomized studies on intervention effects. In: Higgins JPT, Thomas J, Chandler J, Cumpston M, Li T, Page MJ and Welch VA (eds) Cochrane handbook for systematic reviews of interventions version 6,1 (updated September 2020) (Cochrane)

Reimers MS, Bastiaannet E, Langley RE, van Eijk R, van Vlierberghe RLP, Lemmens VEP, van Herk-Sukel MPP, van Wezel T, Fodde R, Kuppen PJK, Morreau H, van de Velde CJH, Liefers GJ (2014) "Expression of HLA class I antigen, aspirin use, and survival after a diagnosis of colon cancer", Jama. Intern Med 174:732-739

Restivo A, Cocco IMF, Casula G, Scintu F, Cabras F, Scartozzi M, Zorcolo L (2015) Aspirin as a neoadjuvant agent during preoperative chemoradiation for rectal cancer. Br J Cancer 113:1133-1139

Schwarzer G (2015) Meta-analysis with R. Cham, Heidelberg, New York, Dordrecht, London, Springer

Stang A (2010) Critical evaluation of the newcastle-ottawa scale for the assessment of the quality of nonrandomized studies in metaanalyses. Eur J Epidemiol 25:603-605

R Core Team (2019) "R: A language and environment for statistical computing". R Foundation for Statistical Computing, Vienna, Austria. https://www.R-project.org/

Walker AJ, Grainge MJ, Card TR (2012) Aspirin and other non-steroidal anti-inflammatory drug use and colorectal cancer survival: a cohort study. Br J Cancer 107:1602

Weitz J, Koch M, Debus J, Hohler T, Galle PR, Buchler MW (2005) Colorectal cancer. Lancet 365:153-165

Wu S, Gan Y, Wang X, Liu J, Li M, Tang Y (2013) PIK3CA mutation is associated with poor survival among patients with metastatic colorectal cancer following anti-EGFR monoclonal antibody therapy: a meta-analysis. J Cancer Res Clin Oncol 139:891-900

Zanders MMJ, van Herk-Sukel MPP, Vissers PAJ, Herings RMC, Haak HR, van de Poll-Franse LV (2015) Are metformin, statin and aspirin use still associated with overall mortality among colorectal cancer patients with diabetes if adjusted for one another? $\mathrm{Br} \mathrm{J}$ Cancer 113:403

Zell JA, Ziogas A, Bernstein L, Clarke CA, Deapen D, Largent JA, Neuhausen SL, Stram DO, Ursin G, Anton-Culver H (2009) Nonsteroidal anti-inflammatory drugs effects on mortality after colorectal cancer diagnosis. Cancer 115:5662-5671

Publisher's Note Springer Nature remains neutral with regard to jurisdictional claims in published maps and institutional affiliations. 